\title{
Determination of Activity of Multiple Sclerosis (MS) Lesions in the Brain by Different Magnetic Resonance Imaging (MRI) Pulse Sequences
}

\author{
HOSAM A. YOUSEF, M.D.*; ANWER M. ALI, M.D.**; NISREEN A. ABBAS, M.D.* and \\ SARA A. ABUEL-WAFA, M.Sc.* \\ The Departments of Diagnostic Radiodiagnosis* and Neurology**, Faculty of Medicine, Assiut University, Assiut, Egypt
}

\begin{abstract}
Background: Multiple sclerosis is one of the commonest causes of chronic neurological disability in young and middle aged people. MRI offers a unique in-vivo investigative tool for its evaluation.

Aim of Study: To reveal the value of different MRI pulse sequences in evaluation of multiple sclerosis affection of the brain.

Patients and Methods: This is a prospective hospital analytic study enrolling 25 patients diagnosed to have definite multiple sclerosis according to the McDonald's Criteria for diagnosing multiple sclerosis during the period from April 2016 to February 2017. The study was conducted in the MRI Unit of the Radiology Department of Assiut University Hospital.

Results: All the enhancing lesions showed restricted diffusion in DW images at high $\mathrm{b}$ values accounting for $100 \%$ sensitivity and negative predictive value of DWI pulse sequence. On the other hand, some of the non-enhancing MS lesions, showed diffusion restriction in DW images, assuming a positive predictive value and specificity of $36.3 \%$ and $94 \%$ respectively. Post-contrast Magnetization Transfer Contrast (MTC) images could significantly detect higher number of enhancing MS lesions than standard T1W-SE images. All the enhancing lesions depicted on post-contrast $\mathrm{T} 1 \mathrm{~W}$-SE sequence were also seen enhancing in MTC images, but more conspicuous.

Conclusion: Diffusion weighted imaging and magnetization transfer imaging provide suitable investigative tools for determination of multiple sclerosis activity.
\end{abstract}

Key Words: Multiple sclerosis - Active lesions - Diffusion weighted imaging - Magnetization transfer contrast.

\section{Introduction}

MULTIPLE sclerosis is one of the commonest causes of chronic neurological disability in young

Correspondence to: Dr. Hosam A. Yousef, E-Mail: hosameldeen67@yahoo.com and middle aged people. The understanding of the disease has advanced considerably over the last two decades due to the development of Magnetic Resonance Imaging (MRI). MRI enabled noninvasive in-vivo visualization of the pathological stages of the disease in the living brain over its course [1]. It also provided better understanding of the mechanism of neuronal injury and the potential of certain treatment lines in neuronal repair

MRI has proven to be far superior to CT in the diagnosis of white matter lesions due to its sensitivity to white matter changes, higher spatial resolution and multiplanar capabilities. The demyelination plaques are characterized initially by an abnormal increase in water content (edema), followed by disruption of the blood-brain barrier, perivascular inflammatory reaction and eventually myelin loss. The focal increase in water content produces hyperintense signal in the affected foci, thus making visualization even of small plaques quite easy. Conventional MRI pulse sequences including dual-echo T2-weighted (T2W), fluidattenuated inversion recovery (FLAIR) and postcontrast T1-weighted (T1W) sequences are useful not only in the diagnostic workup of patients in whom MS is suspected, but also to measure the burden of the disease and to monitor the disease course in patients with confirmed MS. Other MR techniques proved to have higher pathological specificity, including magnetization transfer contrast imaging, diffusion weighted imaging (DWI), and proton-spectroscopy. These MR techniques have been extensively applied to measure the disease burden at different stages of the disease 
DW imaging is currently routinely implemented as part of the protocol of MRI in MS patients, and has been proposed as an adjunct for screening disease activity in MS [4]

MTC imaging improves the contrast between MS lesions and Normally Appearing White Matter (NAWM), both in Spin-Echo (SE) and GradientEcho (GE) sequences [5]. The resulting selective signal suppression can be used for both lesion characterization by means of a magnetization transfer ratio [6] and for contrast augmentation of enhancing lesions [7,8]. MTC has been successfully applied to post-contrast T1W images to increase detection of active MS lesions [5].

\section{Aim of study:}

The aim of this study is to demonstrate the value of different MR pulse sequences in determination of activity of MS lesions in the brain.

\section{Patients and Methods}

This prospective hospital analytic study was conducted during the period from April 2016 to February 2017.

\section{Inclusion criteria:}

- Patients known to have multiple sclerosis and presented with symptoms and signs of current activity.

- All the patients fulfilled the McDonald's criteria for diagnosing multiple sclerosis.

\section{Exclusion criteria:}

- Presence of contraindication for MRI as presence of cardiac pacemakers, intracranial metallic surgical clips or retained metallic foreign bodies.

- Patients with known allergy to Gadolinium contrast media.

Twenty-five were included in the study. The patients had median age of 35 years (range 15-50 years), and a median Expanded Disability Status Scale (EDSS) of 4. There were 22 female and 3 male patients. They were grouped into three different clinical subgroups according to the pattern of clinical presentation; 20 patients with relapsingremitting MS, 3 with secondary progressive MS and 2 with primary progressive MS. The median duration of disease was 2 years (range 1 to 5 years).

The study was conducted in the MRI Unit of the Radiology Department of Assiut University Hospital. The patients were recruited from the outpatient clinic and inpatient ward of the Center for Neurology and Neurosurgery of Assiut University Hospital. The clinical data including the findings of neurological examination as well as the results of relevant investigations for each patient were obtained.

Among the 25 patients, 6 patients were presented with visual disturbances, 6 with ataxia, 4 with motor symptoms, 3 with hearing loss, and 4 patients were presented with sensory symptoms. There was no significant time gap between the occurrence of clinical symptoms suggesting disease activity, and performing MRI examination. None of the examined patients had contraindication for MRI examination.

\section{Ethical considerations:}

All the study procedures were done confidentially after approval of the Ethical Committee of the Faculty of Medicine in Assiut University. An informed consent was obtained from each patient included in this study.

\section{MRI protocol:}

All MRI examinations were performed on a 1.5 Tesla MRI system (Achieva, Philips Medical Systems, Best, The Netherlands) using a 16-channel phased-array SENSE-head coil according to the following protocol; a fast-field multi-planar SMART-BRAIN localizer survey was initially obtained, upon which all subsequent pulse sequences were planned. A calibration "reference" scan was then obtained to allow the use of SENSE and CLEAR options that increase the image signal-tonoise ratio and improve image quality. The following standard pulse sequences were obtained; sagittal T1W, coronal T2W-TSE and axial FLAIR, dualecho T2W-TSE and EPI-DWI. Post-contrast study was done for all patients using Gd-DTPA (Magnevist, Scherring, Germany) which was administered intravenously at a dose of $0.1 \mathrm{mmol} / \mathrm{kg}(7-10 \mathrm{cc})$. Post-contrast sagittal, coronal and axial T1W images were obtained. Axial MTC sequence was also done after contrast in all patients.

\section{MR imaging analysis:}

MS lesions which show enhancement in postcontrast T1W-SE images and/or MTC images were identified. Also, MS lesions showing restricted diffusion in DW images were identified. MS lesions were classified according to its location into white matter lesions (peri-ventricular and subcortical), grey matter lesions (cortical and deep grey matter nuclei), and cerebellar and brainstem lesions. The lesion conspicuity at different pulse sequences was determined. 


\section{Statistical analysis:}

All statistical calculations were done using computer program SPSS (Statistical Package for the Social Science; SPSS Inc., Chicago, IL, USA) Version 21 for Microsoft Windows.

\section{Results}

\section{Determination of MS lesion activity:}

Forty-one MS enhancing lesions were detected in the 25 patients of the study after intravenous contrast. On the other hand, 72 lesions were found showing restricted diffusion in DW images. All the 41 enhancing lesions showed restricted diffusion in DW images at high $b$ values. The anatomical location and function of these non-enhancing restricted lesions were correlated with the relapse in all these patients. Anatomical location and function of the areas affected with such. These findings indicate that the overall active MS lesion detectability in DW images is superior to Gadoliniumenhanced images.

The application of the MTC sequence increased the sensitivity for detection of Gadolinium enhanc-

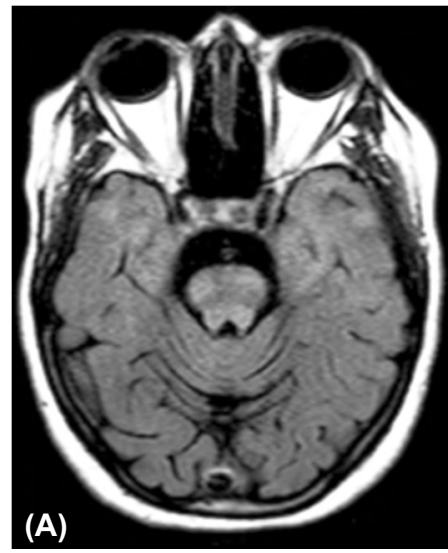

ing lesions; there was no statistically significant difference in the number of detected enhancing lesions between the two pulse sequences. Fortyone lesions have shown enhancement in post-Gad MTC images, among which only 34 lesions showed enhancement in post-contrast $\mathrm{T} 1 \mathrm{~W}$ images. The enhancement is more conspicuous in post-contrast MTC images than the corresponding T $1 \mathrm{~W}-\mathrm{SE}$ images.

Table (1): Number of detected MS lesions.

\begin{tabular}{llll}
\hline & $\begin{array}{c}\text { Gd } \\
+\mathrm{ve}\end{array}$ & $\begin{array}{c}\mathrm{Gd} \\
-\mathrm{ve}\end{array}$ & Total \\
\hline Restricted lesions in diffusion weighted & 41 & 72 & 113 \\
Non-restricted lesions in diffusion weighted & 0 & 1168 & 1168 \\
\hline Total & 41 & 1240 & 1281 \\
\hline
\end{tabular}

Table (2): Sensitivity and specificity of diffusion restriction in DW images and for lesion enhancement.

\begin{tabular}{ll}
\hline Sensitivity & $100 \%$ \\
Specificity & $94 \%$ \\
Positive predictive value & $36.3 \%$ \\
Negative predictive value & $100 \%$ \\
\hline
\end{tabular}

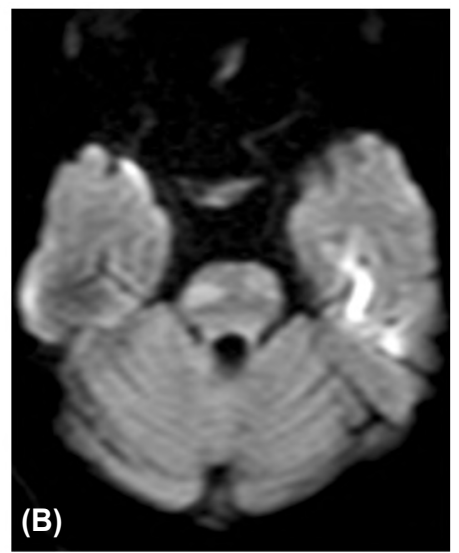

Fig. (1): FLAIR image (A) in an 18 years old female patient known to have remitting-relapsing MS presented with relapse, showing multiple ill-defined faint hyperintense lesions involving the anterior part of the pons on both sides. In DW images (B), only the large lesion in the right side of the pons shows restricted diffusion.
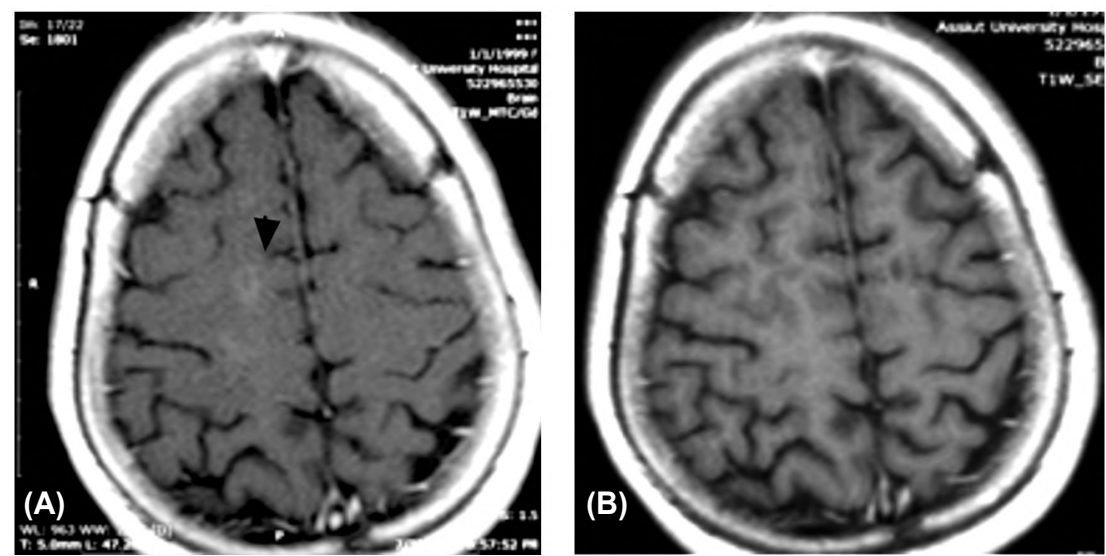

Fig. (2): A ring enhancing lesion (representing activity) is seen in post-contrast MTC (A) (arrow) while poorly visualized in post-Gad T1W images (B). 

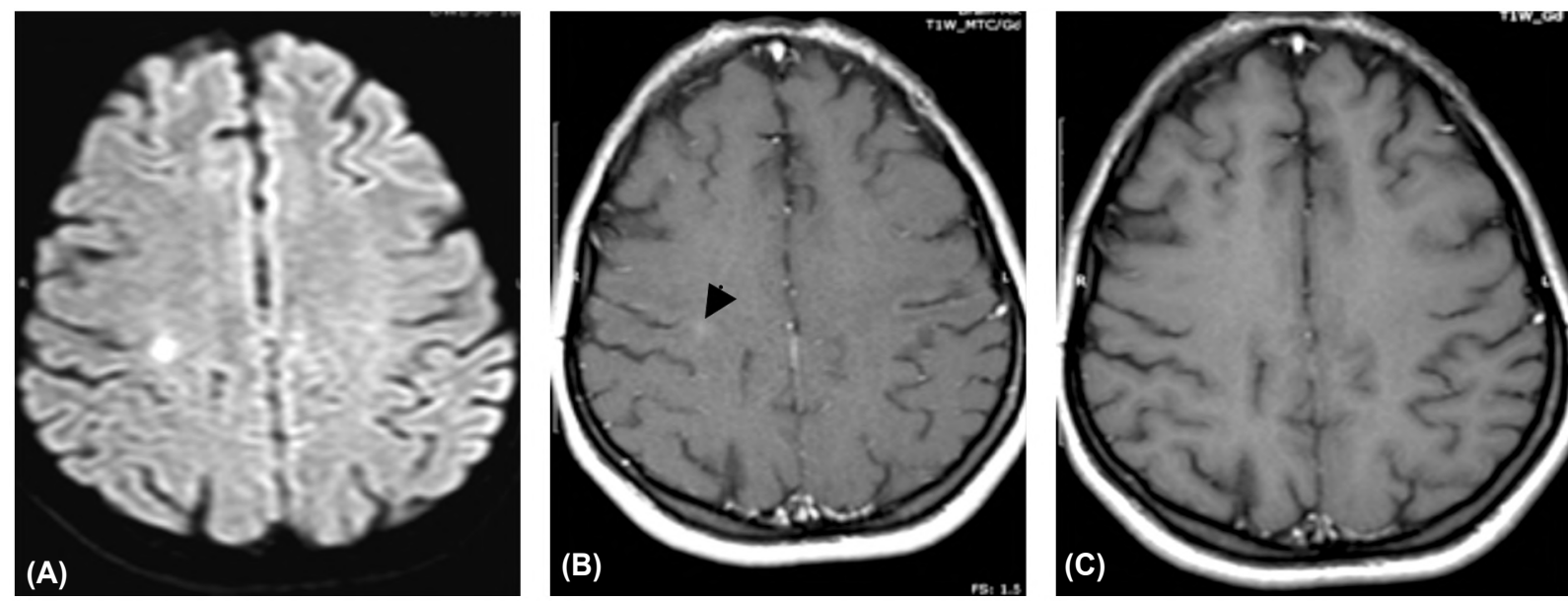

Fig. (3): A right parietal subcortical lesion shows restricted diffusion in DW image (A) in a 21 years old female patient. The lesion shows good enhancement in post-contrast MTC image (B) but not in post-contrast T1W image (C).

\section{Discussion}

One of the most important items in MRI assessment of MS patients is the determination of disease activity. Lesion enhancement after contrast was once considered the corner-stone in determination of lesion activity where enhancement denotes activity. Recent researches have correlated diffusion restriction in MS lesions with lesion activity, especially at high $b$ values; so that active MS lesions show restricted diffusion compared to inactive ones Abou Zeid et al., [9].

The presence of enhancing lesions in MS is an indicator of inflammation and breakdown of the blood-brain barrier. It has been considered the MRI hallmark for monitoring of MS. The inflammatory component of the disease is usually present in earlier stages of MS lesions [10]

Previous studies conducted by Phuttharak et al. and Tievsky et al., have shown that the center of ring-enhancing MS lesions shows facilitated diffusion in contrary to its enhancing edge which displays restricted diffusion [11,12]

Diffusion weighted imaging has contributed to better understanding of the patho-physiological mechanisms of Multiple Sclerosis (MS). The background inhibition in DW images allows the detection of even very small lesions within the brain parenchyma. It also improves the specificity of MR in characterization of different types of MS lesions [13]

In the present study, 41 enhancing MS lesions were detected, all were found to show restricted diffusion in DW images, and all have been accurately correlated with the recent clinical presenta- tion in the patients. This indicates significant link between diffusion restriction and post-Gadolinium enhancement in MS lesions, so that all enhancing lesions showed restricted diffusion in DW images (100\% sensitivity and $100 \%$ NPV). These results are in agreement with those of Lo et al., which found that all 51 enhancing MS lesions showed restricted diffusion in DW images [14].

A Spanish cohort study described $52.63 \%$ sensitivity for DW-MRI in determination of MS lesion activity. However, this might be attributed to a relatively long gap between the onset of symptoms in those patients and the performance of MRI examination (up to 44 days). It is better to perform MRI examination for MS patients shortly after onset of symptoms of relapse (within 1-8 days). It has been described that the diagnostic performance of post-contrast MRI for demonstration of gadolinium-enhancing and lesions is better when performing MRI within the first 30 days of relapse of symptoms [15].

In our study, the time gap between the onset of symptoms and performing MRI was short (2-7 days).

On the other hand, in this study, not all lesions showing restricted lesions in DW images showed enhancement in post-contrast images (36.3\%). Two possible explanations are suggested; first, the lesion hyperintensity in DW images is more sensitive and lasts longer (may persist several months) than lesion enhancement due to the transient disruption in blood-brain barrier (which usually lasts 4-6 weeks). Second, the high signal of some lesions seen in DW images may be attributed to the "T2 shine-through" effect. The signal intensity in DW 
images is influenced by water diffusivity and the intrinsic $\mathrm{T} 2$ properties of the tissue being examined. The increased water content in demyelinating lesions may cause prolongation of $\mathrm{T} 2$ relaxation time rendering high signal on $\mathrm{T} 2 \mathrm{~W}$ images and thus, hyperintensity on DW images. The lack of enhancement in post-contrast images in lesions with restricted diffusion in DW images is likely due to recovery of the blood-brain barrier. The persistent hyperintensity in DW images may also suggest residual extracellular edema with increased diffusion, prolonging $\mathrm{T} 2$ relaxation time, and the $\mathrm{T} 2$ shine-through effect. It also might be due to axonal loss and gliosis with widening of the extracellular space [16,17].

Another possible explanation for absence of enhancement in lesions with restricted diffusion is that those patients had already started steroid pulse therapy for treating the disease relapse. Steroids not only restore the integrity of BBB, but also suppress the inflammatory reaction which is partly responsible for the enhancement in MS lesions.

On the other hand, a study conducted by Abdoli et al., [18] stated that most lesions with restricted diffusion showed enhancement in CE-T1W images (high specificity $\geq 94 \%$ ), but many enhancing lesions showed no evidence of restricted difusion in DW images (low sensitivity $\leq 34 \%$ ).

Magnetization transfer imaging improves the visibility of enhancing multiple sclerosis lesions, because it has higher contrast-to-noise ratio than conventional post-contrast $\mathrm{T} 1 \mathrm{~W}$ images. If magnetization transfer contrast would be used in evaluation of enhancement in MS lesions, a pre-contrast study would be necessary $[\mathbf{5 , 8}]$. In this study, we used magnetization contrast for detection of enhancement in MS lesions, so we performed precontrast MT sequence to be compared with the post-Gadolinium MT images. The results of our study show that post-contrast MT could better detect post-contrast enhancement than conventional post-contrast $\mathrm{T} 1 \mathrm{~W}$ images. This is in agreement with the findings of Mehta et al., and Van Waesberghe et al., [5,8].

\section{Conclusion:}

The current study describes the relative merits of some MRI parameters in their suitability for evaluation of MS affection of the brain. Systematic application and proper knowledge of different MRI techniques is required to elucidate the relationship of the qualitative and quantitative measures derived with evolution of the disease.

\section{References}

1- SMORODCHENKO A., WUERFEL J., POHL E.E., VOGT J., TYSIAK E., GLUMM R., et al.: CNS-irrelevant Tcells enter the brain, cause blood-brain barrier disruption but no glial pathology. European Journal of Neuroscience, 26 (6): 1387-98, 2007.

2- ZIPP F.: A new window in multiple sclerosis pathology: Non-conventional quantitative magnetic resonance imaging outcomes. Journal of the Neurological Sciences, 287: S24-S9, 2009.

3- FILIPPI M., ROCCA M.A., De STEFANO N., ENZINGER C., FISHER E., HORSFIELD M.A., et al.: Magnetic resonance techniques in multiple sclerosis: The present and the future. Archives of Neurology, 68 (12): 1514-20, 2011.

4- YURTSEVER I., HAKYEMEZ B., TASKAPILIOGLU O., ERDOGAN C., TURAN O.F. and PARLAK M.: The contribution of diffusion-weighted MR imaging in multiple sclerosis during acute attack. European Journal of Radiology, 65 (3): 421-6, 2008.

5- VAN WAESBERGHE J., CASTELIJNS J., LAZERON R., A. NIJEHOLT G.L. and BARKHOF F.: Magnetization Transfer Contrast (MTC) and long repetition time spinecho MR imaging in multiple sclerosis. Magnetic Resonance Imaging, 16 (4): 351-8, 1998.

6- DOUSSET V., GROSSMAN R.I., RAMER K.N., SCHNALL M.D., YOUNG L.H., GONZALEZSCARANO F., et al.: Experimental allergic encephalomyelitis and multiple sclerosis: lesion characterization with magnetization transfer imaging. Radiology, 182 (2): 483 91, 1992.

7- FINELLI D.A., HURST G.C., GULLAPALI R.P. and BELLON E.M. : Improved contrast of enhancing brain lesions on postgadolinium, T1-weighted spin-echo images with use of magnetization transfer. Radiology, 190 (2): 553-9, 1994.

8- MEHTA R.C., PIKE G.B. and ENZMANN D.R.: Improved detection of enhancing and nonenhancing lesions of multiple sclerosis with magnetization transfer. American Journal of Neuroradiology, 16 (9): 1771-8, 1995.

9- ZEID N.A., PIRKO I., ERICKSON B., WEIGAND S., THOMSEN K., SCHEITHAUER B., et al.: Diffusionweighted imaging characteristics of biopsy-proven demyelinating brain lesions. Neurology, 78 (21): 1655-62, 2012.

10- GRAZIANO E., HAGEMEIER J., WEINSTOCKGUTTMAN B., RAMASAMY D.P. and ZIVADINOV R.: Increased contrast enhancing lesion activity in relapsing-remitting multiple sclerosis migraine patients. NeuroImage: Clinical, 9: 110-6, 2015.

11- PHUTTHARAK W., GALASSI W., LAOPAIBOON V., LAOPAIBOON M. and HESSELINK J.R.: ADC measurements in various patterns of multiple sclerosis lesions. Journal-Medical Association of Thailand, 89 (2): 196, 2006.

12- TIEVSKY A.L., PTAK T. and FARKAS J.: Investigation of apparent diffusion coefficient and diffusion tensor anisotropy in acute and chronic multiple sclerosis lesions. American Journal of Neuroradiology, 20 (8): 1491-9, 1999. 
13- MASCALCHI M., FILIPPI M., FLORIS R., FONDA C., GASPAROTTI R. and VILLARI N.: Diffusion-weighted MR of the brain: Methodology and clinical application. La Radiologia Medica, 109 (3): 155-97, 2005.

14- LO C.P., KAO H.W., CHEN S.Y., CHU C.M., HSU C.C., CHEN Y.C., et al.: Comparison of diffusion-weighted imaging and contrast-enhanced T1-weighted imaging on a single baseline MRI for demonstrating dissemination in time in multiple sclerosis. B.M.C. Neurology, 14 (1): 100, 2014

15- ROVIRA À., SWANTON J., TINTORÉ M., HUERGA E., BARKHOF F., FILIPPI M., et al.: A single, early magnetic resonance imaging study in the diagnosis of multiple sclerosis. Archives of Neurology, 66 (5): $587-$ 92, 2009.
16- VAN DER VALK P. and De GROOT C.: Staging of multiple sclerosis (MS) lesions: Pathology of the time frame of MS. Neuropathology and Applied Neurobiology, 26 (1): 2-10, 2000.

17- EISELE P., SZABO K., GRIEBE M., ROSSMANITH C., FÖRSTER A., HENNERICI M., et al.: Reduced diffusion in a subset of acute MS lesions: A serial multiparametric MRI study. American Journal of Neuroradiology, 33 (7): 1369-73, 2012.

18- ABDOLI M., CHAKRABORTY S., MacLEAN H.J. and FREEDMAN M.S.: The evaluation of MRI diffusion values of active demyelinating lesions in multiple sclerosis. Multiple Sclerosis and Related Disorders, 10: 97-102, 2016.

\title{
تحديل نشاط التصلب المتعدد بالمخ بالطرق المختلفة للتصوير بالرنين المغناطيسى بالهئي
}

\author{
التصلب المتعدل هو مرض المناعة الذاتية التى تهاجم الإستجابة المناعية الجهاز العصبى المركزى بالجسم حيث يؤثر بشكل إنتقائى مما

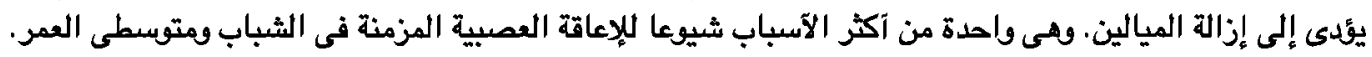

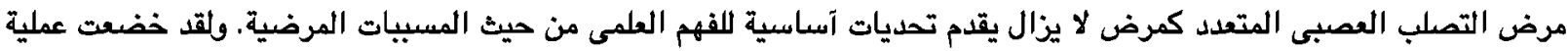

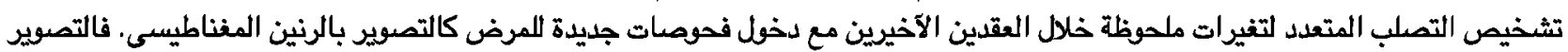

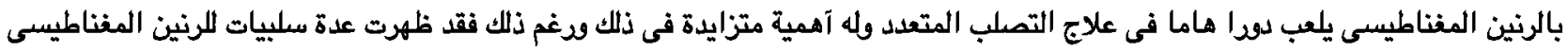

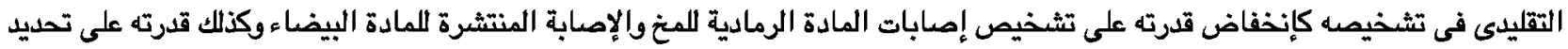

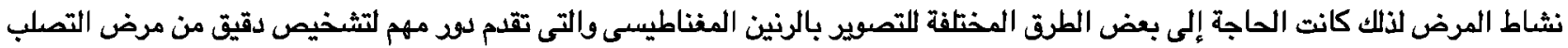 \\ العصبى المتعدد ورصد نثاط المرض. المران.

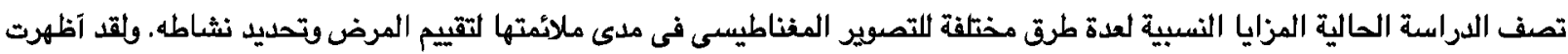

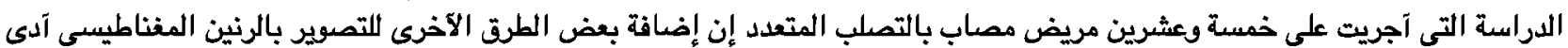

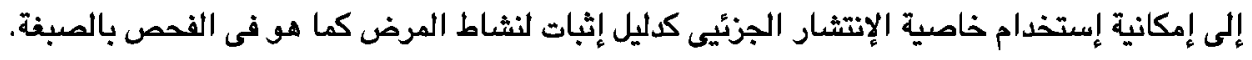

